\title{
Photoelectric Properties of the Ferroelectric Polymer Poly(vinylidene fluoride)
}

\author{
A. V. Bune, V. M. FridKin, K. A. Verkhovskaya, \\ and George TAYLOR* \\ Institute of Crystallography of the Academy of Sciences of the USSR, \\ 117333, Moscow, USSR \\ * Research Center of the Princeton Resources, P.O. Box 211, \\ Princeton, N.J. 08540, U.S.A.
}

(Received October 21, 1988)

\begin{abstract}
The photoconductivity, the bulk photovoltaic effect (BPE) and the photoelectret effect were observed experimentally in ferroelectric polymer films of poly(vinylidene fluoride) (PVDF). The mutual influence of the photoelectret internal field on the hysteresis loops and ferroelectric switching on the photoelectret charge is discussed.

KEY WORDS Ferroelectric Polymer / Bulk Photovoltaic Effect / Photoelectret Effect / Depolarization / Ferroelectric Switching /
\end{abstract}

During the last ten years, the ferroelectric polymers of PVDF and their copolymers were widely investigated. ${ }^{1}$ The task of the present work is the investigation of the photoelectric and photovoltaic properties of ferroelectric polymers. Previously many authors ${ }^{2-5}$ investigated photocurrent and the bulk photovoltaic effect (BPE) in PVDF. The experimental results reported in these works are in discrepancy. The open-circuit saturation voltage for a $30-\mu$ m-thick film was estimated by Sasabe et al. ${ }^{2}$ to be $4 \times 10^{4} \mathrm{~V}$, which exceeds the energy gap $E_{\mathrm{g}}$ of PVDF more than by three orders of magnitude $\left(E_{\mathrm{g}} \simeq 9 \mathrm{eV}\right)$. An analogous result for copolymer VDF-TrFE was obtained. ${ }^{5}$ Ogden and Gookin ${ }^{3}$ measured the open-circuit voltage directly for $27-\mu \mathrm{m}$ thick film and obtained a value $V=$ $0.25 \mathrm{~V} \ll E_{\mathrm{g}}$ Micheron $^{4}$ noted in question the existence of BPE in poly(vinylidene fluoride) and connected these observation with injection of carriers from electrode into PVDF. The discrepancy of results ${ }^{2-5}$ seems to arise from the fact that not the same material was used in different experiments.
The photoelectret effect was not observed in PVDF earlier in spite of the stable thermoelectret effect. ${ }^{6}$ In the present work the photoelectret effect in PVDF was found and investigated.

\section{EXPERIMENTAL}

The ferroelectric PVDF films in this work were supplied by the film "Bamberg". Three types of $14-\mu$ m-thick samples were used: a) uniaxially stretched films with a $\beta$-form crystal structure, b) biaxially stretched films with $\alpha$ and $\beta$-form crystal structure, and c) unstretched films with $\alpha$ and $\beta$ phases. All the films used were poled, but pyroelectric and piezoelectric properties were different according to the concentration of $\beta$-form crystals.

The photoconductivity and BPE were measured by illumination in the visible region. The light source was a $500 \mathrm{~W}$ Xe lamp and Ar laser $(\lambda \simeq 458 \mathrm{~nm})$. The semitransparent gold electrodes were evaporated on the film surfaces. Due to this, the photocurrent and photovoltaic current were measured in the direction of the 
spontaneous polarization. The film was illuminated in the same direction. The absorption coefficient $\alpha^{*}$ was estimated from the optical transmittances for two samples of different thickness. The obtained value was $\alpha^{*} \simeq$ $30 \mathrm{~cm}^{-1}$ in the region $400-600 \mathrm{~nm}$ and therefore the $14-\mu \mathrm{m}$-thick films were illuminated uniformly. The hysteresis loops were recorded under $50 \mathrm{~Hz}$ using the classical Sawyer and Tower method. Transient pyroelectric currents were observed, when the light was turned on or off. The maximum values of pyrocurrents were measured.

\section{RESULTS AND DISCUSSION}

\section{Photoconductivity}

The measured photoconductivity of PVDF $\sigma_{\mathrm{ph}}$ was $1.0 \times 10^{-12} \Omega^{-1} \mathrm{~cm}^{-1}$ for the light intensity $I \simeq 1 \mathrm{~W} \mathrm{~cm}^{-2}$, which is two orders of magnitude greater than the dark conductivity $\sigma_{\mathrm{d}}$. The measurement of photocurrents is obtained when the transient pyroelectric current vanishes to zero. The spectral distribution of the photoconductivity was measured by means of a monochromator and modulated light. Figure 1 shows the spectral distribution of the photoconductivity for $\beta$ phase (type a) and a broad maximum at $\lambda \simeq 600 \mathrm{~nm}$. Thus PVDF shows extrinsic photoconductivity because $E_{\mathrm{g}} \simeq 9 \mathrm{eV}$. Figure 2 shows the photocurrent-voltage characteristic, which reveals the superlinear part, caused by the photocurrent

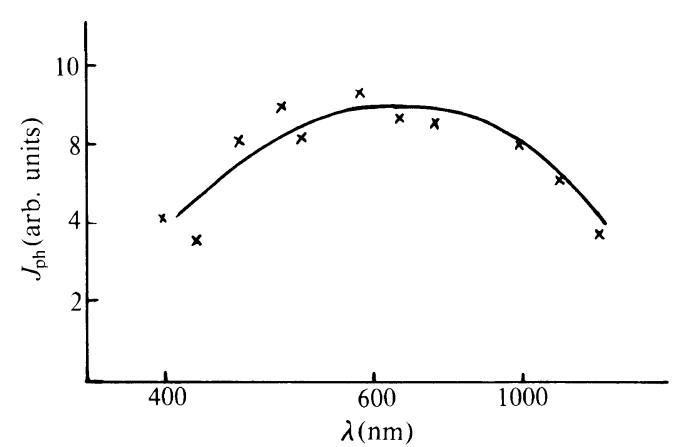

Figure 1. Spectral distribution of the photoconductivity for $\beta$-phase PVDF. limited by the volume charge. The dependences of the photocurrent $J_{\mathrm{ph}}$ and pyrocurrent $J_{\text {pyro }}$ on the light intensity were investigated (Figure 3). The dependence $J_{\text {pyro }}$ (I) is linear in the whole interval of the light intensity. But the dependence $J_{\mathrm{ph}}$ (I) has a superlinear part.

We also investigated the copolymer $\left(\mathrm{CF}_{2}-\right.$ $\left.\mathrm{CF}_{2}\right)_{n}-\left(\mathrm{CH}_{2}-\mathrm{CF}_{2}\right)_{m}$ which showed no photoconductivity. The different photosensitivity of the sample is connected with impurities, introduced in the polymer at the time of synthesis and production.

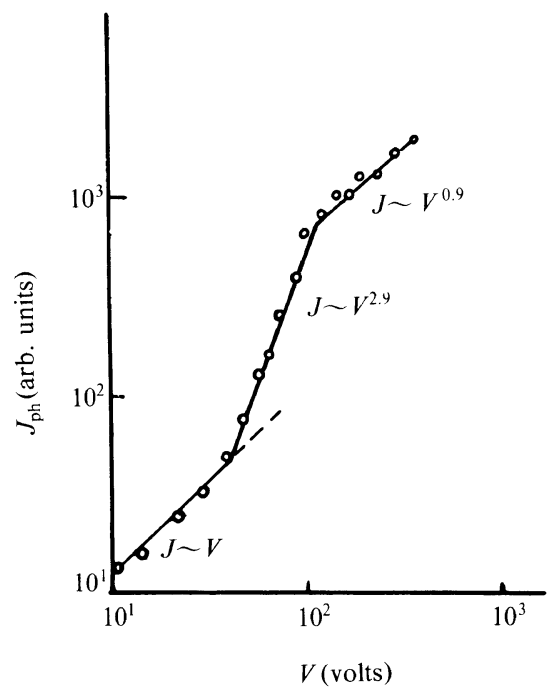

Figure 2. Photocurrent-voltage characteristics for $\beta$ phase PVDF. $\lambda=458 \mathrm{~nm}$

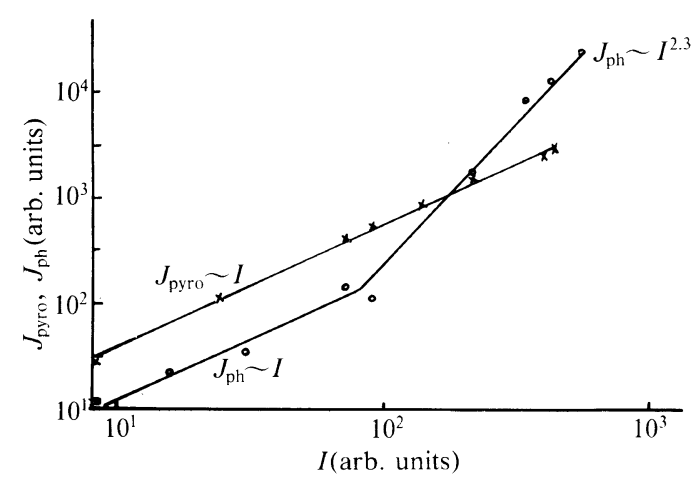

Figure 3. Lux-ampere characteristics of the photocurrent $J_{\mathrm{ph}}$ and pyrocurrent $J_{\text {pyro }}$ for $\beta$-phase PVDF. $\lambda=$ $458 \mathrm{~nm}$. 


\section{Bulk Photovoltaic Effect}

The bulk photovoltaic effect discovered for the first time for ferroelectrics ${ }^{7-9}$ is a particular case of a more general effect discribed for crystals and textures without a center of symmetry by the third-rank tensor $\alpha_{i j k}$

$$
J_{i}=\alpha_{i j k} E_{j} E_{k}^{*}
$$

According to eq 1 , when a homogeneous ferroor piezoelectric crystal (or texture) is illuminated uniformly with linearly polarized light, a photovoltaic current $J_{i}$ arises in it. The sign and magnitude of the current depend on the orientation of the light polarization vector with projections $E_{j}$ and $E_{k}$. Taking into account the point group of the $\beta$-phase of PVDF $(\mathrm{mm} \mathrm{2})$ and the difference from zero components of the photovoltaic tensor $\alpha_{i j k}$, we obtain from eq 1 the expression for $J_{z}$ (the direction of the linear polarized light propagation coincides with $z$-axis)

$$
J_{z}=\alpha_{31} I+\left(\alpha_{32}-\alpha_{31}\right) I \sin ^{2} \theta
$$

where $I$ is the light intensity, $\theta$ is the angle between the light polarization plane and stretching axis and the $z$-axis is perpendicular to the film surface. The expression for $J_{z}$ under illumination with natural light has the form,

$$
J_{z}=k \alpha^{*} I
$$

where

$$
k=\frac{1}{2}\left(\alpha^{*}\right)^{-1}\left(\alpha_{31}+\alpha_{32}\right)
$$

Figure 4 shows the kinetics of the shortcircuit photocurrent generated in the poled sample (type a) when the light is switched on and off. The surface of the film was illuminated

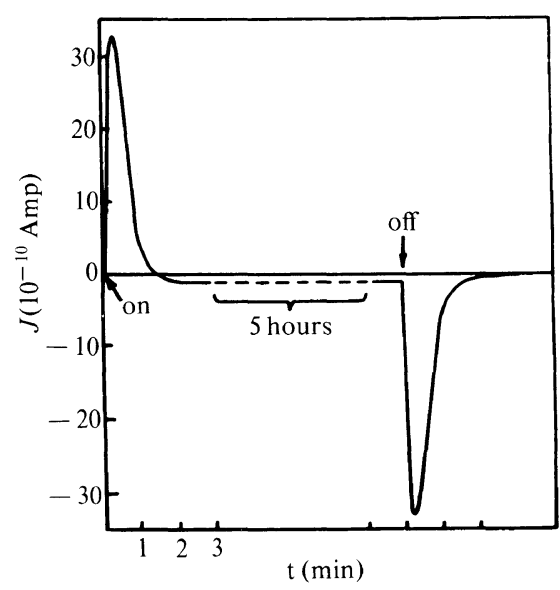

Figure 4. Kinetics of the photoresponce in the $\beta$-phase for short-circuited electrodes. $I=1.4 \mathrm{Wcm}^{-2}$.

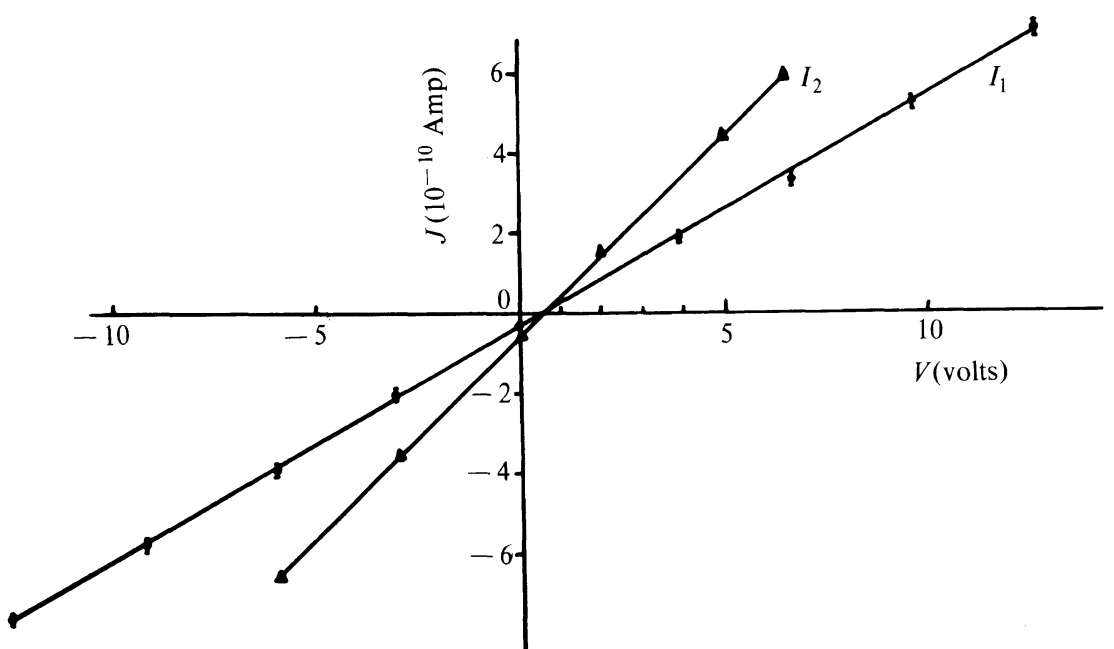

Figure 5. Photocurrent-voltage characteristics of the $\beta$-phase for two different light intensities. $I_{1}=$ $1.4 \mathrm{Wcm}^{-2}, I_{2}=1.8 \mathrm{Wcm}^{-2}$. 
with natural light. The stationary bulk photovoltaic current $J_{z}$ was observed during 5 hours. The value of $J_{z}$ linearly depends on the light intensity $I$. The ferroelectric switching of the sample leads to change of the sign of $J_{z}$. The substitution of $J_{z} \simeq 7.5 \times 10^{-10} \mathrm{~A} \mathrm{~cm}^{-2}$, $I \simeq 1.4 \mathrm{~W} \mathrm{~cm}^{-2}$, and $\alpha^{*} \simeq 30 \mathrm{~cm}^{-1}$ into eq 3 gives $k \simeq 2 \times 10^{-11} \mathrm{~A} \mathrm{~cm}(\mathbf{W})^{-1}$. For "Kureha" films, $k \simeq 1.0 \times 10^{-13} \mathrm{Acm}(\mathrm{W})^{-1} .^{3}$ The same dependence was observed in the films of types b and c. However, the magnitudes of the photovoltaic current were smaller and thus $k<10^{-11} \mathrm{Acm}(\mathrm{W})^{-1}$ due to the smaller concentration of th $\beta$ phase.

Figure 5 shows the photocurrent-voltage characteristics of the $\beta$ phase (type a). It is seen from Figure 5 that the open-circuit photovoltage for $14-\mu \mathrm{m}$-thick film is equal to $0.6 \mathrm{~V} \ll E_{\mathrm{g}}$. There is no contradiction between this result and existence of BPE. The relationship between the short-circuit $J_{z}$ and the open-circuit voltage $V$ is given by eq $4^{7}$

$$
V=\frac{J_{z}}{\sigma_{\mathrm{ph}}+\sigma_{\mathrm{d}}} l
$$

where $\sigma_{\mathrm{ph}}$ and $\sigma_{\mathrm{d}}$ are the photo- and dark conductivity respectively. $l$ is the distance between the electrodes. The measured value of the photovoltage is in good agreement with the estimated one from eq 4 . According to eq 4, $V$ is proportional to $l$ and may be larger than the energy gap $E_{\mathrm{g}}$ for thick films.

The above mentioned results do not reveal the microscopic nature of the BPE in PVDF. The ferroelectric polymer is a semicrystalline nonhomogeneous material. Therefore, for microscopic explanation of BPE in PVDF the model which was applied to the ferroelectric ceramics may be used. The BPE in the ferroelectric ceramics is connected with the composition of the elementary photo e.m.f. which develops on separate microcrystals. ${ }^{10}$

\section{Photoelectret Effect}

The possibility of photoelectret polarization in ferroelectrics is based on photoconductivity

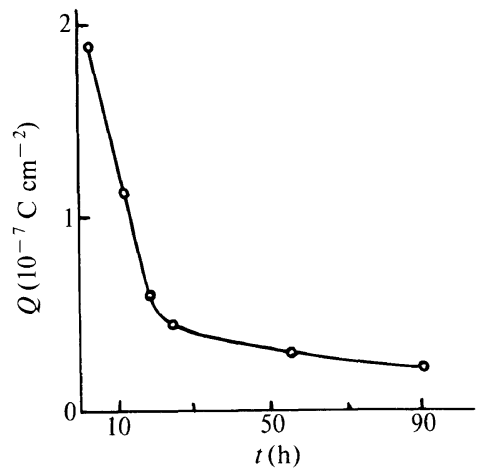

Figure 6. Kinetics of the photoelectret dark depolarization for $\beta$-phase PVDF.

and deep traps. The stability of photoelectret polarization is determined by a large value of $u / k T$, where $u$ is the energy of the traps responsible for the photoelectret effect. ${ }^{11}$

It was found that photoconductivity in PVDF leads to volume charge, which remains for a long time in the poly(vinylidene fluoride) film after switching off the light and electric field. The kinetics of the photoelectret dark depolarization are shown in Figure 6 for type a sample. The curve of the photoelectret charge decay consists of rapid and slow parts. The relaxation time of the rapid part $\tau_{1} \simeq 10^{5} \mathrm{~s}$.

Experiments were performed with $14-\mu \mathrm{m}$ thick film of the $\beta$-phase (type a). The values of the spontaneous polarization $\mathrm{P} \simeq 5 \mu \mathrm{C} \mathrm{cm}^{-2}$ and coercive field $E_{\mathrm{c}} \simeq 0.7 \mathrm{MV} \mathrm{cm}^{-1}$ were obtained from the hysteresis loops. Figure 7 shows curves of the photoelectret polarization and depolarization. The sequence of observation and measurements was the following. The constant voltage $V=600 \mathrm{~V}$ was applied to the film, which was illuminated through the semitransparent electrode in the visible region. The photocurrent was observed at the time of illumination, which decreased due to the volume charge (Figure 7, curve 2). The sample was polarized during 1 hour, after which the illumination was switched off and the external field was removed and the electrodes were shortcircuited. After 1 hour, the polarized sample was illuminated for the second time. 


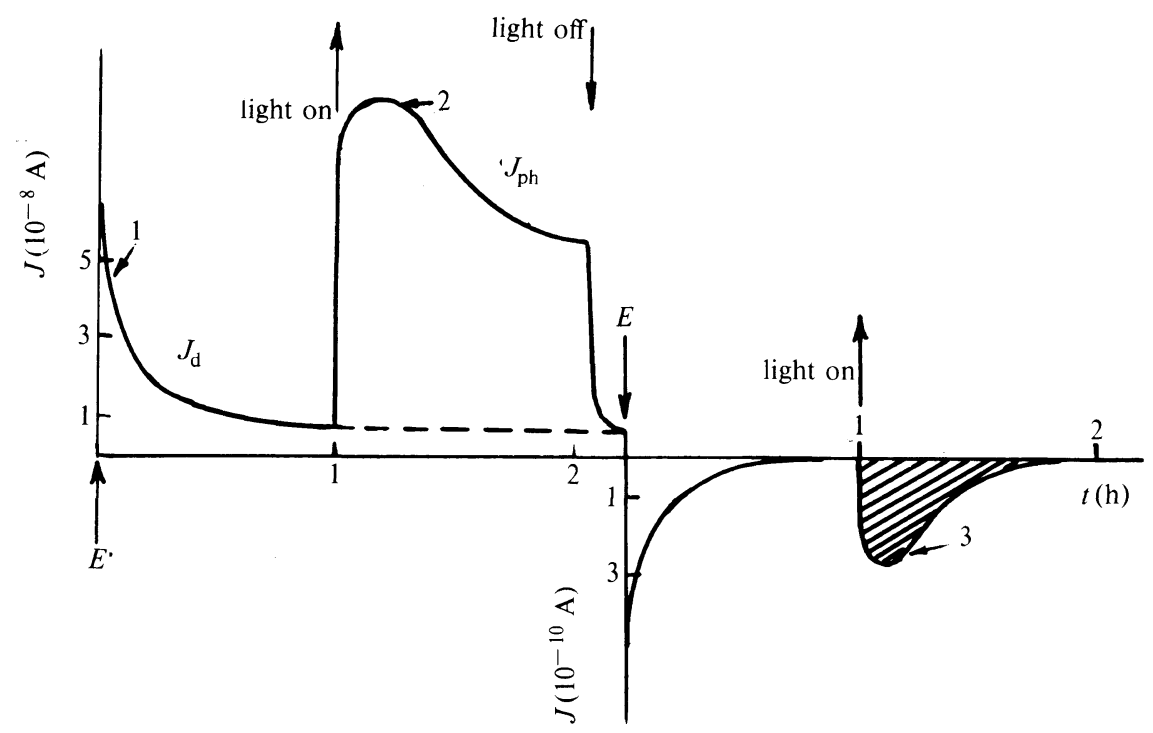

Figure 7. Kinetics of the photopolarization and photodepolarization of the photoelectret for $\beta$-phase PVDF. At the time of light switching, there are transient pyrocurrents, which are not shown in the figure.
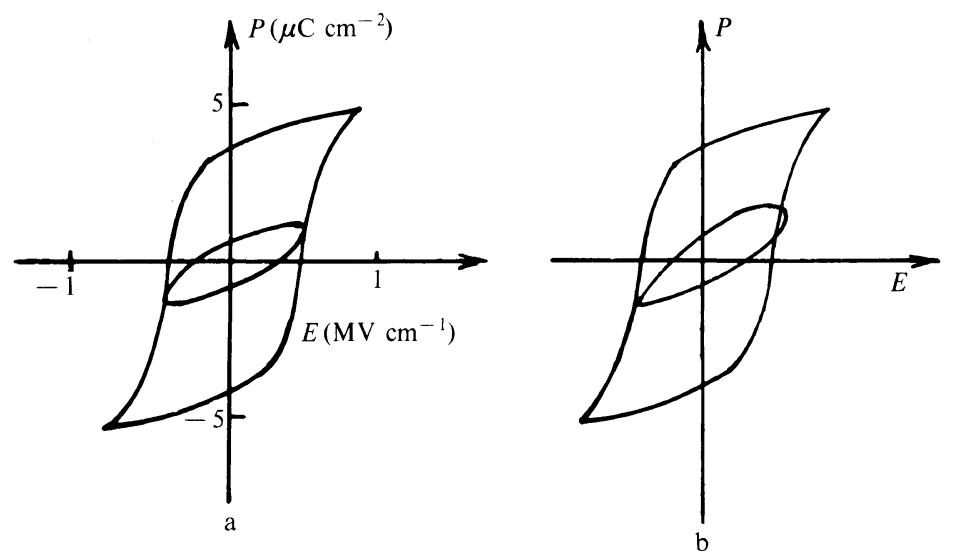

Figure 8. Ferroelectric hysteresis loops before (a) and after (b) photoelectret polarization.

The illumination leads to the photodepolarization current (Figure 7, curve 3). By integrating the photodepolarization current over time, the density of the photoelectret charge $Q \simeq$ $8 \times 10^{-7} \mathrm{C} \mathrm{cm}^{-2}$ was determined. This value corresponds to the internal field of the photoelectret $E \simeq Q / \varepsilon \varepsilon_{\mathrm{o}} \simeq 0.9 \mathrm{MV} \mathrm{cm}^{-1}$ which is the same order of magnitude as the coercive field.

\section{Influence of the Photoelectret Internal Field on} the Ferroelectric Switching

Figure 8 shows the influence of the photoelectret internal field on the ferroelectric hysteresis loop of PVDF. Figure 8a shows the typical symmetric hysteresis loop in the PVDF film without photoelectret polarization. It is seen from Figure $8 \mathrm{~b}$ that after creation of photoelectret polarization, the hysteresis loop becomes asymmetric. The change of sign of the 
photoelectret polarization reverses the asymmetry of the loop. The depolarization of the PVDF photoelectret at illumination leads to recovery of the symmetric loop. Thus, the asymmetry of the hysteresis loop is caused by the internal field of the photoelectret. This phenomenon was observed earlier in the ferroelectrics of the $\mathrm{A}_{\mathrm{V}} \mathrm{B}_{\mathrm{VI}} \mathrm{C}_{\mathrm{VII}}$ type. $^{11}$

Influence of the Ferroelectric Switching on the Charge of the PVDF Photoelectret

The observation of the ferroelectric hysteresis loops in the PVDF photoelectrets revealed decrease of the photoelectret charge during ferroelectric switching. This is seen from Figure 8, which shows the transition of the asymmetric loop in the symmetric one during the ferroelectric switching. The transition time $\tau$ depends on the amplitude of the switching field. In the low electric fields $E<E_{\mathrm{c}}$, $\tau$ is a few seconds. In the electric fields $E \geqslant E_{\mathrm{c}}$ $\tau \lesssim 10^{-3} \mathrm{~s}$ and coincides with the switching time. ${ }^{1}$ It was shown that the decrease of the photoelectret charge is directly connected with the ferroelectric switching. This is illustrated in the Figure 9, which shows the dependence of the photoelectret charge density $Q$ on the amplitude of the alternating field $E(50 \mathrm{~Hz})$. In the low electric field $E \lesssim 10^{-1} \mathrm{MV} \mathrm{cm}^{-1}$ the photoelectret charge $Q$ is not changed. For these fields, there is no hysteresie loop and correspondingly the dependence of polarization on field is linear. In the field region

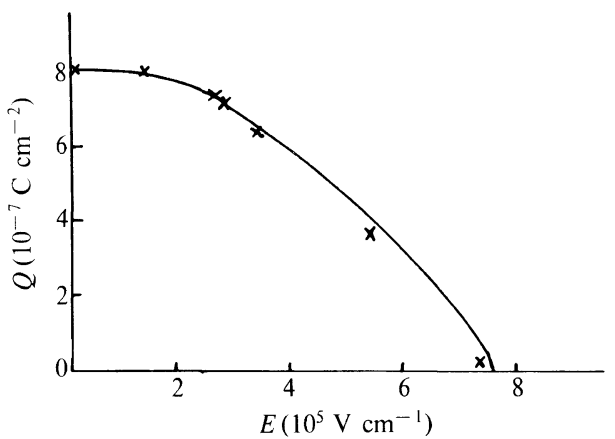

Figure 9. Change of the photoelectret charge in the external alternating field.
$10^{-1}<E<0.3 \mathrm{MV} \mathrm{cm}^{-1}$ the nonsaturated hysteresis loops are observed and correspondingly there is decrease of the charge $Q$. In the high fields $\mathrm{E} \gtrsim E_{\mathrm{c}}$ the saturated hysteresis loops are observed and correspondingly $Q \simeq 0$. The heating of the sample in the alternating field was negligibly small.

In spite of the observation of the asymmetric hysteresis loops in ferroelectric photoelectrets, their depolarization in the alternating electric fields was not observed. ${ }^{11}$ Perhaps this new phenomenon is specific for ferroelectric polymers. Here we propose a model for this phenomenon.

Let us consider first the mechanism of the formation and dark relaxation of the photoelectret polarization in the $n$-type crystals. ${ }^{12}$ The energy-band diagram is shown in Figure 10. Here $M_{1}$ and $M_{2}$ are the concentrations of the donors and traps respectively, $N$ is the concentration of electrons in the traps, $\beta$ and $\alpha$ are the probabilities of trapping and recombination and $k^{T}$ is the probability of the electron thermal excitation from the trap to the band:

$$
k^{T}=\beta N_{\mathrm{c}} \exp (-u / k T)
$$

where $N_{\mathrm{c}}=2\left(2 \pi m k \dot{T} / h^{2}\right)^{3 / 2}$ is the density of states, $u$ is the trap energy and $m$ is the effective mass of electron.

The illumination of the crystal in the external electric field $E$ leads to filling of the traps by electrons and their redistribution in the crystal volume. This leads in turn to the crea-

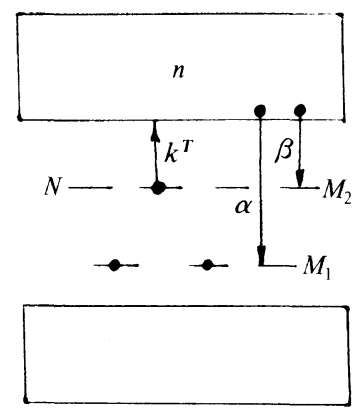

Figure 10. Energy-band diagram of the photoelectret. 
tion of the photoelectret charge $Q$. Provided that $d \ll 1$, where $d$ is the screening length and $l$ is the length of the crystal in the field direction (the photoelectret of the barrier type), $Q$ has the form

$$
Q=\int_{0}^{d} \rho(x) \mathrm{d} x
$$

where $\rho$ is the volume charge density.

The dark depolarization of the photoelectret is caused by the thermal excitation of the trapped electrons and their recombination (Figure 10). If the trapping is small in comparison with the recombination $(\beta / \alpha<1)$, the kinetics of the photoelectret dark depolarization are exponential. ${ }^{12}$

$$
\ln \frac{Q_{\mathrm{o}}}{Q}=k^{T} t
$$

where $Q_{0}$ is the initial value of the charge and $t$ is the time of the dark depolarization. The time of the dark relaxation $\tau=\left(k^{T}\right)^{-1}$ is determined by the trap energy $u$.

In ferroelectrics, $u$ can be expanded in a series in the spontaneous polarization $P{ }^{7}$

$$
u_{1}=u_{2}+\frac{a_{1}}{2} P^{2}+\cdots
$$

The possible mechanism of the influence of the ferroelectric switching on the photoelectret charge relaxation is described by eq 5,7 , and 8 . For effective switching time $P=0$ and hence the energy of the traps decreases for the value $\Delta u=u_{1}-u_{2}$ which in turn accelerates the depolarization of the photoelectret.

Substitution of the typical values $N_{\mathrm{c}} \simeq$ $10^{19} \mathrm{~cm}^{-3}$ and $\beta \simeq 10^{-8} \mathrm{~cm}^{3} \mathrm{~s}^{-112}$ in $\tau=\left(k^{T}\right)^{-1}$ gives

$$
\tau=10^{-11} \exp (u / k T)
$$

The value of $\Delta u$ can be obtained from eq 9 . The substitution of the dark relaxation time $\tau_{1} \simeq 10^{5} \mathrm{~s}$ in eq 9 gives $u_{1} \simeq 0.9 \mathrm{eV}$. The switching time in PVDF for the coercive field $E_{\mathrm{c}} \simeq 0.8 \times 10^{6} \mathrm{MV} \mathrm{cm}^{-1}$ is $\tau_{2} \simeq 10^{-3} \mathrm{~s}^{1}$ This value of the switching time can be taken as the

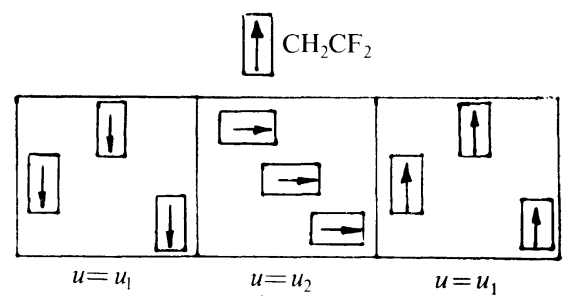

Figure 11. Influence of the ferroelectric switching on the trap energy in PVDF.

effective time of relaxation of the photoelectret charge in th external alternating field $E=E_{\mathrm{c}}$. Substituting $\tau_{2}=10^{-3} \mathrm{~s}$ in eq 9 we obtain $u_{2} \simeq 0.5 \mathrm{eV}$ and hence $\Delta u \simeq 0.4 \mathrm{eV}$. In PVDF $P \simeq 5 \mu \mathrm{Ccm}^{-2}$ and in accordance with eq 8 $a_{1} \simeq 2 \times 10^{10} \mathrm{eV} \mathrm{cm}^{4} \mathrm{C}^{-2}$. The constant $a_{1}$ (which is proportional to the square of the constant of the electron-phonon interaction) exceeds the value of $a_{1}$ in ferroelectric crystals by two orders of magnitude.

This discrepancy can be explained by the fact that the band theory of the photoelectret is not applicable to the polymer. It may be supposed that for PVDF there is strong dependence of $u$ on the orientation of the dipoles $\mathrm{CH}_{2} \mathrm{CF}_{2}$. This situation is shown schematically in Figure 11. This strong dependence leads to thermal release of carriers from the traps at ferroelectric switching.

\section{REFERENCES}

1. T. T. Wang, J. M. Herbert, and A. M. Glass, Ed., "The Applications of Ferroelectric Polymers," Blackie, Glasgow and London, 1988.

2. H. Sasabe, T. Nakayama, K. Kumazawa, S. Miyata, and E. Fukada, Polym. J., 13, 967 (1981).

3. T. R. Ogden and D. M. Gookin, Appl. Phys. Lett., 45, 995 (1984).

4. F. Micheron, Appl. Phys. Lett., 47, 67 (1985).

5. S. Nakashima, S. Taki, T. Horiuchi, and K. Matsushigo, Rep. Prog. Polym. Phys. Jpn., 30, 507 (1987).

6. G. M. Sessler, Ed., "Electrets," Springer, Berlin, Heidelberg, New York, 1980.

7. V. M. Fridkin, "Photoferroelectrics." Springer, Berlin, Heidelberg, New York, 1979.

8. A. M. Glass, von der Linde, and T. Negran, J. Appl. Phys. Lett., 24, 233 (1974). 
A. V. BUNE et al.

9. V. M. Fridkin, A. A. Grekov, and A. I. Rodin. Ferroelectrics, 43, 99 (1982).

10. V. B. Sandomirskii, Sh. S. Khalidov, and E. V. Chensky, Ferroelectrics, 43, 147 (1982).

11. V. M. Fridkin, "Ferroelectric Semiconductors,"
Consultants Bureau, New York and London, 1980. Chapter 7.

12. V. M. Fridkin, "The Physics of the Electrophotographic Process," Focal Press, London, New York, 1973, Chapter 1. 